Cahiers « Mondes anciens »

MONDES

ANCIENS

Histoire et anthropologie des mondes anciens

$2 \mid 2011$

Journées doctorales ANHIMA 2008 et 2009

\title{
La place de l'editor dans la procession des jeux
}

\section{Antoine Gailliot}

\section{OpenEdition}

Journals

Édition électronique

URL : http://journals.openedition.org/mondesanciens/634

DOI : 10.4000/mondesanciens.634

ISSN : 2107-0199

Éditeur

UMR 8210 Anthropologie et Histoire des Mondes Antiques

\section{Référence électronique}

Antoine Gailliot, « La place de l'editor dans la procession des jeux », Cahiers « Mondes anciens » [En ligne], 2 | 2011, mis en ligne le 20 juillet 2011, consulté le 19 avril 2019. URL : http:// journals.openedition.org/mondesanciens/634; DOI : 10.4000/mondesanciens.634

Ce document a été généré automatiquement le 19 avril 2019.

\section{(c) (i) $\odot$}

Les Cahiers «Mondes Anciens » sont mis à disposition selon les termes de la licence Creative Commons Attribution - Pas d'Utilisation Commerciale - Pas de Modification 4.0 International. 


\title{
La place de l'editor dans la procession des jeux
}

\author{
Antoine Gailliot
}

1 Un passage célèbre de Denys d'Halicarnasse nous renseigne sur la composition et l'ordre de la procession d'ouverture des jeux à Rome, la pompa circensis, qui menait les dieux et les hommes du Capitole au Cirque Maxime ${ }^{1}$. Ce texte complexe a déjà été très souvent commenté, notamment la difficile question des sources utilisées par Denys pour sa description, et celle non moins délicate d'une possible origine étrusque de la cérémonie. Je passe rapidement sur ces aspects et en reprenant ces lignes, je voudrais simplement approfondir un point de détail qui revêt néanmoins une importance capitale dans la détermination de l'ordre de marche et des enjeux civiques et religieux de la procession. Où se trouvait l'editor, le magistrat chargé de l'organisation des jeux? La lecture habituelle et les traductions courantes du début du chapitre 72 font supposer qu'il se trouvait en tête de la procession, et cette opinion est si communément répandue, qu'à ma connaissance, aucun savant n'a pensé le placer ailleurs ${ }^{2}$. Or, une lecture attentive du texte de Denys m'incite à proposer une autre hypothèse : l'editor se trouvait après les cohortes de iuvenes, les athlètes, les ludions, les victimes sacrificielles et les musiciens. Il se trouvait en revanche devant la multitude des statues des dieux portées sur les épaules des -

citoyens ou sur des fercula. En effet, cette disposition est non seulement conforme à la lettre du texte de Denys, mais elle est cohérente avec ce que nous savons des caractéristiques de la pompa circensis et de ses similitudes avec la procession triomphale. Surtout, cet ordre de marche est confirmé par les commentaires sévériens des Jeux séculaires qui nous donnent une image très précise de la mise en place de la procession d'ouverture des jeux honoraires, après les sacrifices à Apollon et à Diane le troisième jour des cérémonies. Enfin, donner à l'editor sa juste place dans le cortège permet de comprendre le risque d'instauratio et d'envisager la cérémonie à la fois dans sa dimension rituelle et dans ses enjeux politiques.

2 L'essentiel de la description de Denys ne pose pas de difficulté particulière, si l'on veut bien faire abstraction des digressions visant à prouver le caractère grec des éléments 
constitutifs de la procession. Denys s'émerveille de cette fastueuse et exubérante altérité qui se déploie devant lui et cherche à la replacer dans les cadres explicatifs qu'il connaît, c'est à dire ceux de l'hellénisme. La thèse centrale des Antiquités Romaines trouve ici un cadre privilégié, et si certains éléments sont décidément irréductibles au monde grec, il faut au moins qu'ils soient étrusques. Denys, selon ses propres dires, se fonde sur Fabius Pictor pour donner une description des jeux votifs d'Aulus Postumius voués lors de la guerre latine en 499 avant notre ère 3 . Même en admettant que Denys suive effectivement Fabius Pictor et qu'il lui soit fidèle, il est plus que probable que ce dernier ait donné une description plus proche des processions des jeux du cirque telles qu'elles avaient lieu à la fin du $\mathrm{III}^{\mathrm{e}}$ siècle avant notre ère plutôt que de celles du début du $\mathrm{v}^{\mathrm{e}}$ siècle. De la même façon, Denys a certainement été influencé autant par ses lectures que par ce dont il a pu être témoin lors de son séjour à Rome. Regardons le cortège : « les enfants des Romains [... ] ouvraient la marche", puis venaient les chars et leurs conducteurs, les athlètes, des danseurs en armes, des satyres, les musiciens, les «porteurs de vases [...] à l'usage des sacrifices", et enfin les "statues des dieux élevées sur les épaules des citoyens». Ce passage est d'une fausse précision, car il donne de la procession une image qui parait complète et cohérente alors qu'en réalité Denys sélectionne les informations afin de renforcer sa thèse. Ce point est important, puisqu'en cherchant à comprendre le mécanisme de la cérémonie, nous devons tenter de la reconstruire et de définir les éléments essentiels de sa constitution. Autant dire que «la» pompa que Denys nous présente est surtout «une» pompa, parmi toutes celles possibles, et qu'il ne faut pas considérer sa description comme exhaustive et définitive ${ }^{4}$.

3 La première difficulté consiste à déterminer qui se trouvait en tête du cortège processionnel. Dans la mesure où ce court passage a engendré beaucoup de confusions, je reproduis le texte de Denys avant d'en proposer une nouvelle lecture :

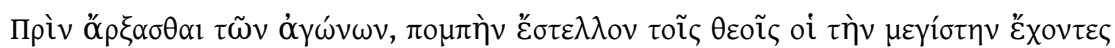

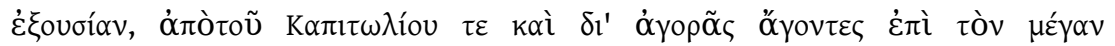

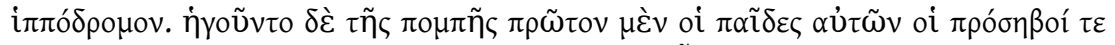

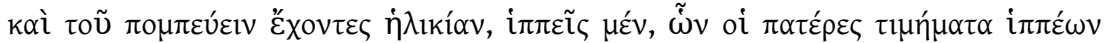

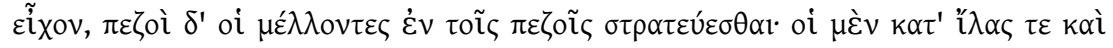

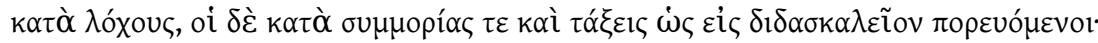

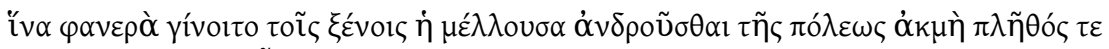

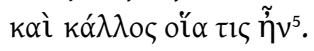

La lecture habituelle est que les premiers magistrats de Rome «menaient » la procession, et on en déduit qu'ils ouvraient la marche. C'est sur ces quelques mots que se concentre le problème, et c'est sur leur fondement que l'on place habituellement l'editor devant tous les autres participants. Je propose au contraire de comprendre, par cette phrase introductive, non pas qu'ils se trouvaient physiquement en tête du cortège, mais qu'ils étaient responsables de son organisation, chargés de la bonne conduite de la cérémonie, dans la mesure où immédiatement après, Denys indique explicitement que les enfants des

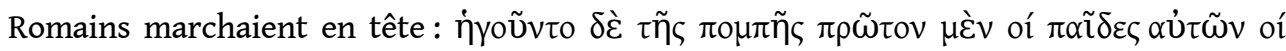

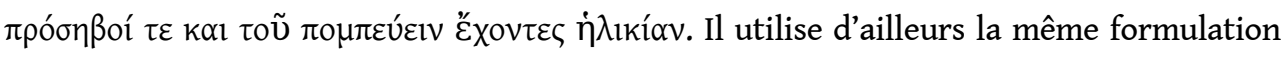
dans un autre passage des Antiquités romaines en parlant des jeunes garçons, זрóønßor

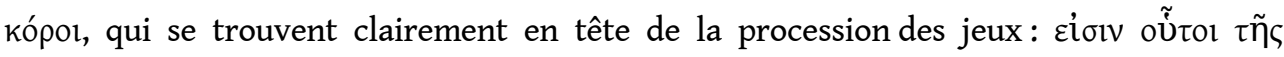

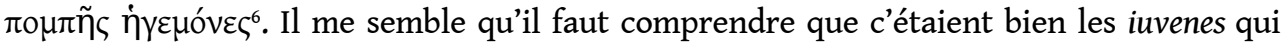
marchaient en premier, les fils des Romains qui approchaient de l'âge adulte, «qui avaient l'âge de participer à la procession, les fils de chevaliers allant à cheval alors que ceux qui étaient destinés à servir dans l'infanterie allaient à pied», devant les 
conducteurs de chars, les athlètes et les ludions, les musiciens, les prêtres et les images des dieux. Si l'on accepte ce raisonnement, il faut bien alors admettre que Denys ne mentionne pas la place exacte de l'editor et de ses clients. Mais force est de constater qu'il ne mentionne pas non plus les victimes sacrificielles, qui devaient pourtant bien êtres conduites en procession, puisqu'à peine arrivées au grand cirque, elles étaient sacrifiées. Parmi la multitude des statues de dieux, il ne parle que de celles juchées sur les épaules des citoyens au moyen de brancards (fercula), sans mentionner les chars ornementés ( tensae), qui étaient justement conduits par des enfants de haute naissance accompagnés de leurs pères. Souvenons-nous que Denys a un programme: mettre en évidence les éléments communs aux pratiques grecques et romaines. Il écarte ou omet ce qui ne lui convient pas ou plus simplement ce qui n'est pas directement utile à sa démonstration.

5 La deuxième difficulté consiste maintenant à placer l'editor dans le cortège processionnel. L'édile qui donnait et présidait les jeux depuis au moins 367 était entouré de ses clients et des autres magistrats ${ }^{7}$. Où se trouvait-il, s'il n'était pas devant? Nous disposons à ce propos d'un faisceau d'indices ${ }^{8}$. Juvénal indique que devant l'editor se trouvaient des musiciens, et que le cortège des Quirites dont il s'était assuré la fidélité par des sportules marchait derrière lui ${ }^{9}$. Surtout, il établit que l'editor (un préteur à l'époque de Juvénal) portait la même tenue que le général victorieux au cours du triomphe, la tunica Iouis, était comme lui juché sur un char, le visage passé au rouge minium, ressemblant en tout point à la statue de Jupiter au Capitole ${ }^{10}$. Cette ressemblance n'est pas le signe d'une incarnation de la divinité ou d'une iconisation du magistrat mais traduit simplement, comme le montre John Scheid, qu'editor et triumphator servent de support à un certain nombre de traits évoquant sans ambiguïté la présence de Jupiter et de sa souveraineté ${ }^{11}$. Les similitudes entre le triomphe et la pompa circensis n'ont pas échappé aux commentateurs Mommsen, Piganiol et Versnel, entre autres : la tenue «étrusco-jovienne » portée par l'editor et le général, les groupes de danseurs, armés ou non, et le renversement des codes de comportement, autorisant la licence et la moquerie. À partir de ce constat, Mommsen a avancé l'idée que la pompa circensis n'était au départ qu'une extension de la pompa triumphalis dans le cadre de la même cérémonie ${ }^{12}$. Afin de fonder son hypothèse, il a proposé une lecture particulière de Tite-Live de façon à établir le caractère initialement votif et non annuel des jeux ${ }^{13}$. Néanmoins, Versnel a montré de façon décisive que la tradition littéraire n'explicite pas de relation directe entre le triomphe et les jeux; que pendant les premiers siècles de la République, les jeux ne furent jamais organisés conjointement à un triomphe et que les jeux votifs établis par notre documentation n'étaient pas fondés sur une victoire militaire célébrée par un triomphe ${ }^{14}$. Il conclut que, malgré des similitudes dans le cérémonial, les jeux ne faisaient pas partie des célébrations triomphales, quelle que soit la période de l'histoire romaine considérée. Cette conclusion n'a pas été remise en cause depuis et est largement acceptée par la communauté scientifique, mais à défaut d'origine commune, les similitudes restent valides et le développement parallèle des cérémonies liées au triomphe d'une part et aux ludi d'autre part au cours de la République se conçoivent difficilement sans influence réciproque, tant les enjeux et les acteurs sont proches.

6 Dans le cas du triomphe, dont la composition et l'ordre de marche sont bien connus ${ }^{15}$, le général victorieux ne se trouve pas en tête de cortège, mais derrière le butin, les prisonniers et les victimes sacrificielles. Il est escorté de ses proches et de ses appariteurs. Derrière lui marchent les soldats, couronnés et porteurs de branches de laurier. Si nous suivons ce modèle dans le cas de la pompa circensis, l'editor pourrait se trouver de la même 
façon derrière les cohortes de iuvenes, les conducteurs de chars, les athlètes, les ludions, les musiciens et les victimes sacrificielles, mais devant les images des dieux. Cette position au cœur du cortège est un pivot essentiel du mécanisme processionnel. À la vitalité des jeunes, à la bravoure des danseurs, à l'énergie de la fête, de la farce et du spectacle, répond la puissance de la cité en ordre, représentée par ses magistrats, ses prêtres et ses dieux. Les danses burlesques, la dérision, l'outrance, permettent la transition entre la partie civique du cortège et la partie sacrée, celle où défilent les dieux portés sur des fercula, leurs attributs placés sur les tensae, ce long cortège que nous rappelle Ovide : voici Victoire, Neptune, Mars, Apollon, Minerve, Cérès et Bacchus/Liber Pater, les Castors et Vénus, auxquels il faut ajouter au moins Consus, Jupiter et Junon, parfois la Magna Mater ${ }^{16}$.

7 Cette césure entre divertissement et solennité, entre exubérance et ordre, différencie également les éléments de la procession qui se trouvent dans la sphère de la rigueur rituelle, de l'obligation religieuse, où l'on ne doit pas trébucher, fléchir sous le poids des imagines, où le pas des victimes sacrificielles ne doit pas hésiter; de ceux qui sont libérés de cette contrainte et dont la vitalité se transmet aux nombreux spectateurs massés tout au long du parcours processionnel jusqu'au cirque. C'est ce que nous dit très précisément Cicéron ${ }^{17}:$ " qu'un tibicen se taise soudainement ou qu'un enfant lâche le cordon du char, le rite n'aura pas été accompli comme ils se doit, et il faut recourir à l'instauratio ». Les accents des musiciens, derrière les satyres, permettent d'ailleurs de prévenir la foule de l'arrivée de l'editor et des dieux, la partie de la procession où l'observance scrupuleuse de la forme est impérative, mais aussi de couvrir les cris, les bruits de toutes sortes, par définition incontrôlables au cœur de la liesse populaire, mais qui provoqueraient des erreurs ou même une interruption ${ }^{18}$.

Iuvenes, conducteurs de chars, athlètes, danseurs, ludions, musiciens, porteurs des objets nécessaires aux sacrifices, victimes sacrificielles, l'editor et son entourage, constitué des magistrats, des membres des collèges sacerdotaux et de ses clients, et enfin la longue théophorie des images divines : cet ordre de marche, fondé à la fois sur une lecture attentive des textes et sur une analyse du mécanisme de la cérémonie, est le plus probable 19. Grâce au témoignage des commentaires épigraphiques des Jeux séculaires, il devient une certitude. Retrouvés en 1890 et 1930 à l'extrémité occidentale du Champ de Mars à Rome, les commentaires augustéens et sévériens sont à l'heure actuelle, malgré leur état fragmentaire, une des sources les plus précises et les plus importantes pour l'étude des rites de la religion romaine ${ }^{20}$. Que nous apprennent ces documents exceptionnels? En 17 avant notre ère, Auguste, avec l'aide du collège sacerdotal des quindécemvirs dont il était le magister, mis en œuvre une séquence rituelle complexe de la mi-mai à la mi-juin. Les prêtres distribuèrent d'abord des torches de soufre et d'asphalte aux citoyens afin de purifier les maisons, puis ces derniers apportèrent aux quindécemvirs des contributions en nature, de blé, d'orge et de fèves, destinées à récompenser les participants aux Jeux. Ces opérations prirent une quinzaine de jours. Puis, dans la nuit du 31 mai au $1^{\mathrm{er}}$ juin, au nom des quindécemvirs et pour le peuple romain, Auguste, assisté d'Agrippa, initia une série de sacrifices nocturnes et diurnes se déroulant pendant trois nuits et trois jours sur le Champ de Mars près du Tibre, au Capitole et au Palatin, en l'honneur d'une part des Moires, des Ilithyes et de Terra Mater, et d'autre part de Jupiter, Junon et enfin Apollon et Diane. Les sacrifices nocturnes furent célébrés selon le rite grec, les victimes, noires, étant entièrement consumées, et ils furent suivis de jeux scéniques et de banquets symboliques offerts aux divinités par cent dix matrones issues des meilleures familles. $\mathrm{Au}$ 
cours de la deuxième journée, une supplication fut adressée à Junon Reine par les matrones, sous la conduite d'Agrippa. Au cours de la troisième journée, un chant, l'hymne séculaire, fut adressé à Apollon et à toutes les divinités honorées pendant les Jeux par deux chœurs, l'un de vingt-sept garçons et l'autre de vingt sept jeunes filles ${ }^{21}$. À l'issu de ces rites, des jeux honoraires, jeux scéniques et jeux du cirque, furent offerts au peuple de Rome pendant sept jours. Les quindécemvirs décidèrent de faire graver dans le marbre la liste des décisions prises et des actes conduits aux cours de ces cérémonies. Ces commentaires furent ensuite installés sur le Champ de Mars, près du Tibre, à l'endroit même ou à proximité immédiate du lieu où les sacrifices nocturnes avaient été offerts. Les commentaires des quindécemvirs relatifs à la célébration de 204 organisée par l'empereur Septime Sévère, retrouvés au même endroit, décrivent une cérémonie globalement comparable, tout en apportant quelques précisions importantes, malgré leurs nombreuses lacunes. Ils sont en effet plus «bavards » que leur homologues augustéens (c'est une évolution nette de l'épigraphie du $\mathrm{III}^{\mathrm{e}}$ siècle, que l'on constate également dans les actes des Frères Arvales), et nous fournissent des indications précieuses sur le détail des rites accomplis, notamment sur le rôle exact des participants à une procession d'ouverture de jeux du cirque, le troisième jour de cérémonies.

Tout d'abord, la ligne 19 du fragment II, qui se trouve dans un passage des commentaires reproduisant un édit des quindécemvirs, établit explicitement que Septime Sévère et ses fils, avec les autres quindécemvirs, sont les organisateurs des jeux : «quos edituri sumus ». Ils sont donc dans la même position institutionnelle que l'édile ou le préteur organisateurs de jeux moins exceptionnels. Plus loin, les actes sévériens détaillent l'ordre de marche des participants à la procession des deux chœurs du Palatin au Capitole, qui passe par la Via Sacra, le Forum, puis l'arc de Septime Sévère (fragment Va, lignes 71-75) :

[ 66 Inde XVviri duxerunt] et tibi [cinum fidicinum 17 cornic] inum aenatorum et tubicin [u] $m$ translatum, et togatorum [8] I [8 a] sinariorum [52] $m$ et tiro [n] umque ludionum quadr [igarum 15 binarum et bi] garum binarum item desultorum cu [rsor] umque factionu [ $\mathrm{m} 9$ singularum] | [pompam] publicis et $c$ [alatoribus 30 ordinantibus adstante et i] ntercede [nte popul] o per [Via] m Sacram Forumque Romanu [m] Arcum Seue [ri et Antoni] ni Aug [[g et 11 Getae Caes (aris)]] pueros pue [[llas]]que [fr] equentes [qui aderant] | [praesi] des praece [debant et publici 27 pompam $t$ [ralatumque tegebant impp seuer [us] et antoninus aug [[g et geta caes (ar)]] cum pr (aefecto) pr (raetorii) sub [sequente] s pueros puellasque sustinente [pom] pa in capitolio cum [ceteris XV / uiris con] uenerunt in [area ante aedem 15 Iou] is [O (ptimi)] M (aximi).

10 Les lacunes et les restitutions parfois hasardeuses de Pighi rendent le commentaire de ce passage difficile. Il n'est pas possible, dans le cadre de cet article, de rendre compte et de résoudre tous les problèmes. Néanmoins, l'interprétation générale ne fait aucun doute: ce déplacement processionnel permet la mise en place de la pompa d'ouverture des jeux du cirque qui vont avoir lieu immédiatement après sur le Champ de Mars, au Trigarium. Pighi propose de lire dans la lacune de 84 lettres en $\mathrm{V}^{\mathrm{a}}$, 71: [66 Inde XVuiri duxerunt]. Il paraît pourtant difficile de placer les quindécemvirs en tête de cortège, alors qu'au cours des autres déplacements dans le cadre rituel des Jeux séculaires, ils se trouvent à proximité immédiate du groupe impérial constitué de Septime Sévère, Caracalla, Geta et Plautien le préfet du prétoire (IV, $4-7 ; \mathrm{V}^{\mathrm{a}}, 46 ; \mathrm{V}^{\mathrm{a}}, 53 ; \mathrm{V}^{\mathrm{a}}, 77 ; \mathrm{V}^{\mathrm{a}}$, 90.8). Si l'on rejette cette restitution, qui n'avait pas été proposée par les éditeurs antérieurs du texte ${ }^{22}$, la lecture devient directe et naturelle : une fois les musiciens, les âniers, les ludions et les acrobates, les conducteurs de chars et les athlètes, encadrés par les esclaves publics et les appariteurs, arrivés au pied du Capitole, les deux chœurs d'enfants, suivis par le groupe impérial et les quindécemvirs montent le Clivus Capitolinus jusqu'au temple de Jupiter 
Capitolin devant lequel doit avoir lieu la deuxième exécution de l'hymne séculaire. Le groupe impérial redescend ensuite (laissant les choristes et les matrones célébrer un dernier rite) et s'engage vers le Tarentum à la suite du reste du cortège resté en bas, afin de clôturer les Jeux séculaires et de donner une session de jeux du cirque ${ }^{23}$ :

I] nde Seuerus et Antoni [nus Augg. [[et Geta/Caes (aris)]] $7 \mathrm{cum}$ ] pr (aefecto) $p$ [r (aetorii) e]

$t c$ [eteris process] erun [t] ad ludos saeculares consummando [s] in thea [tro lign] eo.

11 La nature des commentaires épigraphiques et le contexte strictement rituel du déroulement des cérémonies ne laissent aucun doute. Le groupe constitué par les empereurs et leur suite et par les quindécemvirs se déplace derrière le cortège du cirque dont le caractère festif contraste fortement avec le reste des cérémonies. Cette pompa est à sont tour le reflet des autres pompae circenses organisées en prélude aux Jeux Romains et apporte à mon sens un argument définitif à la question de la place de l'editor dans la procession des jeux.

12 Quelles sont les conséquences de ce résultat? Il y a un fonctionnement organique aux cortèges processionnels organisés dans le cadre d'une séquence rituelle. La procession d'ouverture des jeux mène les dieux et les hommes du Capitole au Circus Maximus. Elle est un prélude nécessaire aux sacrifices sur l'autel souterrain de Consus et permet aux participants de rejoindre leurs postes : pulvinar, gradins, ligne départ; son caractère oscille entre rite et spectacle. C'est cette tension entre l'impératif rituel et l'emprise du scrupule religieux d'une part, et la liesse, la fête et les réjouissances d'autre part, qui lui confère ce caractère si particulier que je nomme le «spectacle de la piété ». Le long du parcours et sur les gradins du cirque, la foule se masse et voit passer devant elle la cité qui s'est mise en scène toute entière, enfants, hommes et dieux. Au moment où le cortège entre dans le cirque, Ovide chante : « Mais voici la procession : faites silence et recueillezvous ; c'est l'heure d'applaudir, la procession dorée est arrivée ${ }^{24} »$. Comment comprendre ces deux vers des Amours? La foule doit à la fois respecter le silence religieux, c'est-à-dire scrupuleux (fauete linguis, selon la formule consacrée utilisée au cours des sacrifices), et applaudir à tout rompre dans un bel enthousiasme collectif. Si l'on doit obtenir le silence à un moment donné, c'est que le bruit de la foule, le vacarme de la liesse régnait auparavant. C'est au moment où défilaient les iuvenes, les auriges, les athlètes et les ludions. Puis vient le temps du silence scrupuleux, un silence bruyant car imposé par les musiciens, au moment où survient l'editor et son entourage. Enfin, la liesse peut s'exprimer sans retenue alors que les dieux passent devant les spectateurs et font leur entrée au Grand Cirque.

13 Au terme de cette discussion, deux éléments essentiels sont acquis. D'une part, l'editor organise la procession d'ouverture des jeux, il est responsable non seulement de son bon déroulement rituel, mais également de la réussite du spectacle, celui qui se déploie dans la rue comme celui qui aura lieu dans le cirque. D'autre part, ce sont les iuvenes qui marchent en tête du cortège, et l'editor se trouve au cœur de la pompa, comme le général est au cœur du triomphe. Lui redonner sa juste place, c'est accorder toute sa cohérence rituelle, civique et festive à la pompa circensis. 


\section{BIBLIOGRAPHIE}

BERNSTEIN F. (1998), Ludi publici: Untersuchungen zur Entstehung und Entwicklung der öffentlichen Spiele im republikanischen Rom, Stuttgart.

CLAVEL-LÉVÊQUE M. (1984), L'Empire en jeux. Espace symbolique et pratique sociale dans le monde romain, Paris.

DuPonT F. (1993), «Ludions, Lydioi : les danseurs de la Pompa Circensis. Exégèse et discours sur l'origine des jeux à Rome ", dans Thuillier J.-P. éd., Spectacles sportifs et scéniques dans le monde italico-étrusque, (Collection de l'École Française, vol. 172), Rome, p. 189-210.

FLAIG E. (2003), Ritualisierte Politik. Zeichen, Gesten und Herrschaft im Alten Rom, Göttingen.

GAILLIOT A. (2009), « Une impiété volontaire ? La procession des jeux et le problème de l'instauratio ", dans Hoffmann G. et Gailliot A. éd., Rituels et transgressions de l'Antiquité à nos jours, Amiens, p. 89-96.

PigANiol A. (1923), Recherches sur les Jeux romains, Strasbourg.

PIGHI G. (1965), De ludibus Saecularibus populi Romani Quiritum, libri sex, Amsterdam.

RÜPKE J. (2006), « Triumphator and Ancestor Rituals between symbolic anthropology and magic », Nume 53, p. 251-289.

SCHEID J. (1981), « Le délit religieux dans la Rome tardo-républicaine », dans Le délit religieux dans la cité antique (Collection de l'École Française, vol. 48), Rome, p. 117-171.

SCHEID J. (1986), « Le flamine de Jupiter, les Vestales et le général triomphant, variations romaines sur le thème de la figuration des dieux ", dans Malamoud C. et Vernant J.-P. éd., Corps des dieux, Paris, p. 213-230.

SCHNEGG-KöLHER B. (2002), « Die augusteischen Säkularspiele », Archiv für Religionsgeschichte 1 (2), p. 9-289.

VERSNEL H. (1970), Triumphus, Inquiry into the Origin, Development and Meaning of the Roman Triumph, Leyde.

\section{NOTES}

1. Denys d'Halicarnasse, Antiquités Romaines, VII, 72, 1-14. En l'absence d'édition dans la C.U.F., j'utilise le texte établi par E. Cary pour la collection Loeb (Roman Antiquities, vol. IV, Cambridge/ Londres, 1943).

2. Voir H. Versnel (1970) et F. BERNSTEIn (1998).

3. Denys d'Halicarnasse, VII, 71, 1-2.

4. Voir F. DUPONT (2006), p. 206.

5. Denys, VII, 72, 1 .

6. Denys, II, 71, 4.

7. Date de la création de l'édilité curule. Dans le texte de Denys, il est question des magistrats dotés de l'imperium, puisqu'il prétend décrire les jeux votifs de Postumius. 
8. Voir notamment dans Varron, Lingua Latina, fr. 32 ; Tacite, Annales, II, 83 ; Suétone, Claude, 11 ; Dion Cassius, XLIV, 6 ; XLVIII, 31 ; L, 8 ; LIX, 13 et LXI, 16 ; Tertullien, De spectaculis, 7.

9. Juvénal, $\mathrm{X}, 36-46$ « quid si uidisset praetorem curribus altis/extantem et medii sublimem puluere circi/ in tunica Iouis et pictae Sarrana ferentem/ex umeris aulaea togae magnaeque coronae/tantum orbem, quanto ceruix non sufficit ulla ?/quippe tenet sudans hanc publicus et, sibi consul/ne placeat, curru seruus portatur eodem./da nunc et uolucrem, sceptro quae surgit eburno,/illinc cornicines, hinc praecedentia longi/agminis officia et niueos ad frena Quirites,/defossa in loculos quos sportula fecit amicos ".

10. À propos des similitudes avec la statue du dieu : Servius, ad. Verg. Ecl., X, 27 : triumphantes qui habent omnia Iouis insignia, sceptrum, palmatam - unde ait Iunenalis in tunica Iouis - faciem quoque de rubrica lnlinunt instar coloris aetherii. À propos de la couleur rouge : Servius, ad. Verg. Ecl., VI, 22 : quod robeus color deorum sit: unde et triumphantes facie miniata, et in Capitolio Iuppiter in quadrigis miniatus.

11. J. SCHEID (1986). Contra J. RÜPKE (2006) qui mène une attaque en règle contre ce qu'il considère comme des idées reçues (ancienneté du triomphe, caractère religieux, identification du général triomphant). Voulant à tout prix promouvoir le principe que le triomphe est un des moyens utilisés par une aristocratie compétitive pour contenir ses rivalités internes en fonction de cadres officiels et légitimants, il considère que le général sur son char est une "statue temporaire" et que son visage passé au minium renvoie aux statues honorifiques en terre cuite qu'il considère courantes dès le $\mathrm{III}^{\mathrm{e}}$ siècle avant notre ère. Le triomphe devient un moyen pour le sénat de gérer les enjeux de prestige associés aux statues honorifiques et la cérémonie serait une création de la toute fin $\mathrm{du}_{\mathrm{IV}}^{\mathrm{e}}$ siècle avant notre ère. Quant à la question de l'origine étrusque de la tenue du général triomphant, il la résout par l'idée que la recherche de prestige individuel des élites aristocratiques passait par des formes iconographiques volontairement archaïsantes.

12. Th. MommSEN (1879), « Die Ludi magni und Romani », Römische Forschungen II, p. 42 sq.

13. Tite-Live, I, 35, 9 : sollemnes deinde annui mansere ludi Romani magnique varie appellati. Tout l'enjeu de la discussion réside dans l'interprétation de sollemnes. Si l'on suit Festus, comme le fait Versnel, il faut entendre " annuellement » et non pas " officiellement » : sollemne sacrum as quod omnibus annis praestari debet (Festus, p. 384 Lindsay).

14. La date des premiers jeux directement liés à un triomphe est discutée, notamment à propos de ceux donnés par L. Anicius Gallus en 167 à l'issu de son triomphe sur l'illyrie, que l'on peut considérer comme des jeux votifs, au contraire des Ludi Victoriae Sullanae de 81 et des Ludi Victoriae Caesaris de 45. Voir à ce sujet H. VERSNEL (1970), op. cit., p. 100-115 et M. CLAVEL-LÉVÊQUE (1984), p. 31-32.

15. Pour la période républicaine voir par exemple sur le triomphe de Scipion en 201, Appien, 8 , 66 ; sur le triomphe de Flamininus en 194 : Tite-Live, XXXIV, 52, 2-12; sur le triomphe de PaulEmile en 167 : Diodore, XXXI, 7, 9-12 et Plutarque, Aemilius, 32, 2-34 ; sur le triomphe de Pompée en 61 : Appien, XII, 17, 116-117 et Plutarque, Pompée, 45 ; sur les triomphes de César (en 46 et 45) : Plutarque, César, 55, 1-4; Suétone, César, 37; 42; 51; sur le triomphe d'Octavien en 29: Dion Cassius, LI, 21,2 - 22,3.

16. Ovide, Amours, III, 2, 43-62 : Sed iam pompa venit : linguis animisque favete !/Tempus adest plausus : aurea pompa venit./Prima loco fertur passis Victoria pinnis/huc ades et meus hic fac, dea, vincat amor!/ Plaudite Neptuno, nimium qui creditis undis !/Nil mihi cum pelago; me mea terra capit./Plaude tuo Marti, miles! Nos odimus arma;/pax iuvat et media pace repertus amor/auguribus Phoebus, Phoebe venantibus adsit!/Artifices in te verte, Minerva, manus/ruricolae, Cereri teneroque adsurgite Baccho!/Pollucem pugiles, Castora placet eques!/Nos tibi, blanda Venus,/puerisque potentibus arcu/plaudimus; inceptis adnue, diva, meis/daque novae mentem dominae! Patiatur amari!.

17. Cicéron, De haruspicum responsis, 23 : An si ludius constitit aut tibicen repente conticuit, aut puer ille patrimus et matrimus si tensam non tenuit [...] ludi sunt non rite facti eaque errata expiantur et mentes deorum immortalium ludorum instauratione placantur.

18. À propos de l'instauratio de la procession des jeux, voir A. GaIlliot (2009) p. 89-96. 
19. Tertullien, de spectaculis, 7, souvent cité à ce propos, ne nous est pas d'une grande utilité. Dans sa vindicte, il mélange des éléments venant aussi bien de la pompa circensis, de la pompa theatralis, de la pompa funebris et d'autres déplacements processionnels: Circensium paulo pompatior suggestus, quibus proprie hoc nomen : pompa parecedit, quorum sit in semetipsa probans de simulacrorum serie, de imaginum agmine, de curribus, de tensis, de armamaxis, de sedibus, de coronis, de exuviis. Quanta praetera sacra, quanta sacrificia praecedant, intercedant, succedant, quot collegia, quot sacerdotia, quot officia moveantur sciunt homines illius urbis in que daemoniorum conventus consedit.

20. Pour les commentaires augustéens : CIL I ${ }^{2}$, p. 29 ; CIL VI, $877=$ VI, $32323=$ VI, 32324. B. SCHNEGG -KÖHLER (2002) propose une édition complète des acta. Pour les commentaires sévériens : CIL VI, 32326-32236, commodément édités et commentés dans G. PIGHI (1965).

21. L'hymne séculaire, composé par Horace, a été conservé par la tradition littéraire. Cf. Horace, Odes et Epodes, texte établi et traduit par F. Villentuve, Paris, Belles Lettres, 1964, $7^{\mathrm{e}}$ édition, p. 185-192.

22. E. DieHL (1932), " $\mathrm{Zu}$ den Neuen Acta ludorum saecularium septimorum des Jahres $204 \mathrm{n}$. ch.", Sitzungsber. Der. Preuss. Ak. Der Wissensch., p. 762-791, et RomAneluI P. (1931), Notizie degli Scavi di Antichità (R. Accademia dei Lincei), vol. VII, série VI, fasc. 7-8-9, p. 313-345.

23. G. PIGHI, op .cit. p. 167, 1. Va 76.

24. Ovide, Amours, III, 2, 43-44: "Sed iam pompa uenit: linguis animisque fauete! / Tempus adest plausus : aurea pompa uenit.

\section{RÉSUMÉS}

À Rome, les jeux du cirque commençaient par une procession solennelle qui menaient les hommes et les dieux du Capitole au Grand Cirque. L'importance du rôle qu'y jouait le magistrat chargé d'organiser les festivités, revêtu d'un costume similaire à celui du général triomphant et entouré de ses clients et des autres magistrats, a déjà été bien souligné. En revanche, sa place dans l'ordonnance du cortège reste à définir précisément. En se fondant sur une relecture attentive d'un célèbre passage de Denys d'Halicarnasse et sur le témoignage fondamental des actes épigraphiques des Jeux séculaires sévériens, cet article propose de donner à l'editor sa juste place, loin derrière les cohortes d'adolescents, les athlètes, les ludions, les victimes sacrificielles et les musiciens, juste devant les statues des dieux, de façon à mieux comprendre le fonctionnement de la cérémonie, aussi bien d'un point de vue rituel que politique.

In Rome, a grand procession was held before public games, where Gods and men marched from the Capitol to the Great Circus. Scholars have stressed the fundamental role of the editor, the roman magistrate in charge of the ceremony, dressed remarkably like a triumphing general, and surrounded by his friends, clients and colleagues. A faulty reading of Dionysius of Halicarnassus has led to surmise that the editor led the marching order. Using the Severian acta of the Saecular Games, combined with a careful re-reading of Dionysius, this article proposes to locate the editor behind the youths, athletes, dancers, musicians and sacrificial victims, just before the statues of the gods. Understanding the correct organisation of the pompa circensis allows in turn for a better understanding of the ritual and political stakes of the ceremony. 
INDEX

Mots-clés : processions, pompa circensis, jeux séculaires, editor, instauratio Keywords : saecular games

\section{AUTEUR}

\section{ANTOINE GAILLIOT}

Université Paris 1 Panthéon-Sorbonne, ANHIMA - UMR 8210 